\title{
Multiplicidade de Papéis da Mulher e seus Efeitos para o Bem-Estar Psicológico
}

\author{
Izabel Cristina Possatti ${ }^{12}$ \\ Mardônio Rique Dias \\ Universidade Federal da Paraíba
}

\begin{abstract}
Resumo
Este estudo examina o envolvimento da mulher em uma multiplicidade de papéis e os conseqüentes efeitos para o bem-estar psicológico. Fizeram parte da amostra 132 mulheres que desempenhavam um trabalho pago e o papel de mãe, as quais responderam a um questionário composto por escalas que mensuram a qualidade do papel de mãe e trabalho pago, uma escala que avalia bem-estar psicológico (QSG-12) e uma escala de satisfação com o casamento. Regressões múltiplas foram feitas entre todas as variáveis e a variável critério, o bem-estar psicológico. Nossos resultados estão de acordo com pesquisas anteriores, mostrando que as recompensas a partir de autonomia no trabalho e autoridade de decisão predizem as medidas de bem-estar psicológico para todas as mulheres e a satisfação com o casamento predizem para todas, exceto para mulheres com elevado status sócio-econômico e mulheres com mais de 39 anos.

Palavras-chave: Multiplicidade; papéis; bem-estar psicológico.
\end{abstract}

Women's Multiple Roles and the Effects on Psychological Well-Being

\begin{abstract}
This study examines the effects of women's multiple roles on psychological well-being. The data were taken from a sample of 132 employed mothers who answered a questionnaire composed of three parts: a roles scale, that measures the quality of the two particular roles (paid worker, mother); a General Health Questionnaire (GHQ-12), that measures psychological wellbeing; and a marital satisfaction scale. Multiple regressions were carried out between all the variables and the criterion variable, the psychological well-being. Our results are in agreement with previous reports, showing that rewards from job autonomy and decision-making authority predicted measures of psychological well-being for all women, while marital satisfaction predicted for all women, except for those with high socio-economic status and those older than 39 years.

Keywords: Multiplicity; roles; psychological well-being.
\end{abstract}

Nos últimos anos, muitas pesquisas têm sido realizadas enfatizando vários aspectos referentes a multiplicidade de papéis e suas conseqüências para a saúde física e mental de homens e mulheres (Amatea \& Fong, 1991; Aston \& Lavery, 1993; Barnett \& Rivers, 1998; Barnett, Marshall \& Sayer, 1992; Bullers, 1994; Gore \& Mangione, 1983; Hecht, 2001; Kibria, Barnett, Baruch, Marshall \& Pleck, 1990; Lennon, 1994; Martire, Stephens \& Townsend, 2000; Nelson, Quick, Hitt \& Moesel, 1990; Reifman, Biernat \& Lang, 1991; Stephens, Franks \& Townsend, 1994; Vandewater, Ostrove \& Stewart, 1997). Porém, estas pesquisas foram realizadas em contextos diferentes da nossa realidade, e considerandose o aumento crescente da população feminina no mercado de trabalho formal no Brasil, faz-se necessário avaliar este aspecto do problema.

\footnotetext{
'Endereço para correspondência: Rua Geraldo Costa 820/804, 58038131, João Pessoa, PB, Fone: (83) 247-1352. E-mail: izapossatti@yahoo.com

${ }^{2}$ A realização desta pesquisa foi possível pelo suporte fornecido pela CAPES, órgão este que gostaríamos de agradecer pelo incentivo a pesquisa científica.
}

Especificamente com relação a mulher, o interesse em estudar os efeitos do desempenho em múltiplos papéis, surgiu a medida em que esta foi ingressando no mercado de trabalho em posições antes só ocupadas pelos homens, assumindo assim mais um papel na sociedade e por estas apresentarem um índice de depressão mais elevado que os homens, o que é atribuído as diferenças nos papéis desempenhados pelas mulheres (Almeida \& Kessler, 1998; Gove \& Tudor, 1973), que são rotineiros e incluem atividades mais difíceis de serem controladas e sem valor social, as mulheres que não possuem uma atividade fora do lar estariam restringidas de outras fontes de gratificação, e mesmo com a entrada das mulheres no mercado de trabalho, as condições que estas enfrentam são mais adversas, propiciando assim maiores prejuízos para o seu bem-estar psicológico.

Embora o distress (tensão psicológica caracterizada pela depressão e ansiedade) não seja considerado doença mental, exceto em casos extremos, a importância em estudar suas causas está no custo social ocasionado por ele, seja na diminuição de produtividade no trabalho por aquele que tem dificuldade de dormir durante à noite, 
seja no sistema médico que dispensa grande parte do atendimento a pessoas com sintomas como enxaquecas, dores de estômago e outras formas de indisposições ocasionadas pelo distress. Geralmente, estas pessoas são tratadas de doenças que elas não possuem, e isto é dispendioso, perigoso e sem nenhuma eficácia (Mirowsky \& Ross, 1989).

À medida que as pesquisas foram avançando, muitos mitos têm se diluído diante dos resultados. Verbrugge (1983) encontrou que a atuação em múltiplos papéis beneficia tanto a saúde física quanto o bem-estar psicológico; Rosenfield (1980) confirma com o resultado de sua pesquisa que as mulheres só apresentam um índice mais elevado de depressão quando as mesmas estão desempenhando papéis tradicionais, e que as mulheres casadas que trabalham são mais saudáveis do que as que não possuem um trabalho remunerado.

Para teorias de acumulação de papéis como a hipótese da expansão (Marks, 1977; Sieber, 1974) ao desempenhar vários papéis os indivíduos não acumulam apenas obrigações, mas também passam a desfrutar de privilégios e se as recompensas forem maiores que as preocupações, o mesmo terá ganhos para a sua personalidade favorecendo o bem-estar psicológico, ou seja, é de suma importância considerar a qualidade dos papéis desempenhados, a causa é que os papéis diferem em valor social e em obrigações e privilégios, assim como a proporção de privilégios e obrigações são diferentes para cada ocupante. É importante ressaltar que dentro desta alternativa foram excluídos os papéis que são considerados intrinsecamente ofensivos ou deprivacionais, como o de um prisioneiro, doente ou nas atividades onde as condições de trabalho são por si só geradoras de mal-estar.

Outros autores enfatizam a importância das requisições de papel para dar sentido a vida de uma pessoa (Thoits, 1983; Vandewater, Ostrove \& Stewart, 1997), pois as identidades são sustentadas através dos relacionamentos de papéis, assim quanto mais posições o indivíduo ocupar, maior será sua segurança existencial, o que ele considera de vital importância para o bem-estar psicológico. O conceito do Eu utilizado por ele provém do interacionismo simbólico, onde o Eu se forma na interação com o social, assim representaria um conjunto de identidades discretas, ou seja, o Eu é definido em termos de posições sociais ocupadas. Thoits (1983) considera que estas posições são psicologicamente protetoras, quando aceitas e realizadas em papéis que o indivíduo esteja realmente identificado, e quanto mais valorizada for uma posição, maior o comprometimento do indivíduo e portanto maior o impacto psicológico de seus ganhos ou perdas. Assim, uma personalidade normal e conduta social apropriada é desenvolvida quando o indivíduo reconhece e adota os papéis associados com sua posição.

O grande questionamento é atribuído ao fato de que pessoas que estejam envolvidas em uma multiplicidade de papéis sociais, freqüentemente são confrontadas com vários estressores, esta é a razão porque alguns estudiosos como Goode (1960) pensam ser a multiplicidade de papéis a causa de prejuízos no bem-estar psicológico. Porém, estes estressores (eventos indesejáveis e persistentes) só causarão distress quando atuarem fazendo mudanças negativas nos papéis das pessoas, provocando a diminuição de autoconceitos como domínio e auto-estima, e quando mediadores como suporte e enfrentamento não estiverem presentes ou não se apresentarem adequados (Pearlin, 1989; Pearlin, Lieberman, Menaghan \& Mullan, 1981).

As quatro características sociais básicas do distress que foram delineadas a medida que as pesquisas foram avançando neste campo foram: a) gênero, o índice de distress apresentado pelas mulheres é mais elevado do que os homens; b) casamento, sabe-se que pessoas casadas apresentam um índice mais baixo que pessoas não casadas; c) eventos de vida indesejáveis, quanto mais eventos negativos uma pessoa experimenta maior o seu distress; d) status sócio-econômico, pessoas com status mais elevado apresentam índices mais baixos de distress. Posteriormente, outras características sociais foram sendo acrescentadas como, por exemplo, o fato de ter filhos aumenta o índice de distress (Mirowsky \& Ross, 1989).

No presente trabalho, objetiva-se a compreensão da relação entre o desempenho de múltiplos papéis e o bemestar psicológico de mulheres, mensurando as recompensas e preocupações do papel de mãe e trabalho pago, satisfação conjugal e o índice de bem-estar psicológico da amostra e conhecer as variáveis antecedentes que contribuíram para o índice de bem-estar psicológico.

\section{Método}

\section{Participantes}

Participaram no estudo 132 mulheres da Paraíba que trabalham e têm filhos, com nível de escolaridade a partir do segundo grau. A maioria destas tinham o terceiro grau $\left(71,2^{\circ} \%\right)$, sendo casadas $(82,6 \%)$, com uma média de idade de 39,6 $(\not p=8,0)$. Com referência às profissões/ocupações desempenhadas pelas participantes da pesquisa, constaram profissionais de diversas áreas, desempenhando funções de complexidade variadas (por exemplo, médicas, advogadas, gerentes de loja, professoras, enfermeiras, administradoras, etc.), nos setores público e privado. As características da amostra foram determinadas pela necessidade de mensurar as recompensas e preocupações com os papéis de mãe e trabalho pago. 


\section{Instrumentos}

Os participantes responderam um questionário composto dos seguintes instrumentos:

Escala Qualidade de Papéis. Compreende aspectos positivos e negativos dos papéis de mãe e trabalhadora. Esta foi elaborada a partir de dados colhidos durante entrevistas com mulheres estadounidenses (Baruch \& Barnett, 1986). A mesma foi validada para a população brasileira com 519 mulheres com as mesmas características da amostra deste estudo. Para cada papel, os respondentes foram solicitados a pensar sobre sua situação no momento atual e a indicar, numa escala de 4 pontos, variando de $1=$ Não no todo a $4=$ Extremamente, a extensão que cada um dos ítens é recompensador ou preocupante.

Através de uma análise fatorial dos eixos principais foi encontrado dois fatores para a escala de recompensa do trabalho (poder de decisão com uma variância explicada de $35,3 \%$ e com uma consistência interna de $\alpha=0,87$; suporte de supervisão, variância explicada $6,3 \%$ e $\alpha=0,83$ ), assim como a escala de preocupação do trabalho (condições de trabalho, variância explicada 36,6 \% e $\alpha=0,92$; sobrecarga, variancia explicada de $5,1 \%$ e $\alpha=0,77$ ) e uma estrutura unifatorial para as escalas de recompensa e preocupação de mãe (desenvolvimento e companbia dos filhos, variância explicada $39 \%$ e $\alpha=0,92)$ para a primeira; e para a segunda (sobrecarga e segurança, variância explicada de 39,4\% e $\alpha=0,91$ ) (Possatti \& Dias, 2000).

Questionário de Saúde Geral - QSG-12: Esta é uma versão reduzida do instrumento original (Goldberg, 1972), composta por 12 ítens, sendo que o QSG foi construído como um teste auto-administrado para diagnosticar desordens psiquiátricas menores. Os itens consistem de questões que procuram saber se o respondente experimentou recentemente um determinado sintoma ou comportamento numa escala 4 pontos, variando de $1=$ Não, absolutamente a $4=$ Muito mais do que de costume. $\mathrm{O}$ instrumento foi traduzido para o português por duas pessoas fluentes nos dois idiomas, e validado na população brasileira com uma amostra de 200 sujeitos (78\% mulheres, $22 \%$ homens, média de idade de 34,4, sendo $63,5 \%$ com terceiro grau completo e de diversas profissões). Apresentou consistência interna satisfatória (Alpha de Cronbach 0,87). A estrutura calculada através do LISREL 8.12 (AFC - Análise Fatorial Confirmatória), como mais adequada foi a bifatorial (goodness of fit: $\chi^{2} / \mathrm{gl}$ $=6,0$, RMSE $A=0,16, G F I=0,77$ e $A G F I=0,66)$, ficando composto de um fator de depressão e o outro de ansiedade, cobrindo assim o conceito de tensão ou distress que se pretende avaliar (Possatti, Dias \& Gouveia, 2000).
Satisfação com o Casamento. Utilizou-se uma escala de satisfação com o casamento, onde os respondentes eram solicitados a pontuar numa escala de 10 pontos, variando de $1=$ Totalmente insatisfeita a $10=$ Totalmente satisfeita, como se sentiam em relação ao casamento no presente, em um passado e futuro próximo. Estas medidas foram derivadas da escala Self-Anchoring Ladder apresentada por Cantril (1965, para revisão ver Dias, 1995), em que o sujeito descreve a melhor e a pior vida possível que ele possa imaginar. A confiabilidade foi examinada utilizando o Alpha de Cronbach, que é uma medida de consistência interna de uma escala baseada na média de correlação dos itens dentro da escala, tendo se apresentado satisfatória $(\alpha=0,77)$.

\section{Procedimentos}

Os questionários foram primeiramente aplicados a um pequeno grupo (10 mulheres) para verificar se a linguagem estava inteligível. Como não ocorreram relatos de dificuldade, prosseguiu-se com as aplicações. Três pesquisadoras treinadas realizaram esta atividade onde era enfatizado o anonimato e a confidencialidade dos dados, como também era informado que a participação na pesquisa era espontânea, ou seja, só participaram da pesquisa as pessoas que não tiveram nenhuma objeção, estando portanto de pleno acordo. Diante da necessidade da amostra ter um trabalho remunerado, grande parte do contato e aplicação foram feitos em locais de trabalho, e nestas situações eram dadas as instruções e o questionário era recolhido em um segundo momento, já que na maioria das vezes elas não podiam interromper suas atividades. Também foram aplicados em universidades e nesta situação os sujeitos levavam de dez a quinze minutos para responder e então era recolhido. Os questionários não apresentaram discrepância quanto as respostas dadas entre os diferentes locais de aplicação. Obteve-se um retorno de 141 questionários para 210 aplicados, o que indica um índice de retorno de 67,1\%; dos que retornaram, 8 foram considerados inválidos por apresentarem mais de 3 itens em branco, restando portanto 133. Destes, 1 foi excluído pelo fato de ter dado respostas que foram consideradas como de valores extremos, sendo então classificado como outlier.

\section{Resultados}

Os dados foram analisados através do SPSSWIN (Statistical Package for the Social Sciences). A amostra estudada apresentou uma pontuação média de $1,86(d p=0,53)$ na escala do Questionário de Saúde Geral (QSG-12), o que indica um bom índice de bem-estar psicológico, já que 
quanto mais próximo de 1, maior o bem-estar psicológico e quanto mais perto da pontuação 4 , maior o índice de distress. Foram computadas as médias de satisfação com o casamento para cada sujeito e posteriormente para amostra total, que apresentou uma média de 7,9 ( $d p=$ 1,6), ficando próxima do pólo positivo, apresentando assim, um bom índice de satisfação.

As médias dos itens de recompensa de cada papel foram mais elevadas do que as preocupações. No caso da qualidade do papel do trabalho, as recompensas tiveram uma média de 2,97 ( $d p=0,56)$, enquanto as preocupações apresentaram uma média de 2,44 $(d p=0,70)$. As mulheres relataram os seguintes aspectos como mais recompensadores: ajudar outras pessoas, a oportunidade de aprender novas coisas, ser capaz de tomar decisões por si própria, o senso de realização e competência que você consegue por fazer o seu trabalho, o seu trabalho se encaixa com seus interesses e habilidades. Os aspectos mais preocupantes foram: oportunidades limitadas para o desenvolvimento profissional ou carreira, ter pequena chance para o crescimento que você quer ou merece, a possibilidade de desemprego, o trabalho não usar suas habilidades, o trabalho enfadonho, monotonia e falta de variedade.

No papel de mãe as recompensas também se apresentaram mais elevadas $(m=3,63, d p=0,41)$ do que as preocupações $(m=2,63, d p=0,70)$. Os aspectos relatados como mais recompensadores foram: ajudar suas crianças a se desenvolverem, o prazer que você consegue a partir da realização deles, compartilhar seus bons momentos, ser incluída em suas vidas. Os aspectos mais preocupantes foram: educação ou formação das crianças, a saúde das suas crianças, as pesadas necessidades e responsabilidades, os problemas que eles podem se envolver.

Com o objetivo de verificar quais as variáveis que contribuem para explicar o bem-estar psicológico foi realizado uma Regressão Múltipla por Etapas (Stepwise). A regressão múltipla fornece, principalmente, o coeficiente de correlação múltipla $(R)$ que indica o grau de correlação existente entre as variáveis antecedentes e a variável critério; o percentual de variância explicada $R^{2}$, o Beta que indica o peso relativo de cada variável para a equação de regressão e o teste $F$ (ANOVA) de significância da relação

Tabela 1

Regressão Múltipla para os Fatores do Bem-Estar Psicológico (N=132)

\begin{tabular}{|c|c|c|c|c|c|c|c|c|}
\hline Variável critério & Variáveis & $R$ & $R^{2}$ & $F$ & $\operatorname{Sig}(\mathrm{F})$ & $\beta$ & $t$ & $p$ \\
\hline \multicolumn{9}{|c|}{ Regressão para a amostra total } \\
\hline \multirow{2}{*}{$\begin{array}{l}\text { Bem-estar } \\
\text { psicológico }\end{array}$} & Poder & 0,35 & 0,12 & $F_{(1 ; 131)}=18,479$ & 0,000 & 0,352 & 4,299 & $<0,0001$ \\
\hline & & \multicolumn{7}{|c|}{ Casadas, todas as faixas etárias e de renda } \\
\hline \multirow[t]{2}{*}{$\begin{array}{l}\text { Bem-estar } \\
\text { psicológico }\end{array}$} & $\begin{array}{l}\text { Poder } \\
\text { de decisão }\end{array}$ & 0,41 & 0,17 & $F_{(1,107)}=21,673$ & 0,000 & 0,410 & 4,655 & $<0,0001$ \\
\hline & $\begin{array}{l}\text { Satisfação } \\
\text { com casam }\end{array}$ & $\begin{array}{l}0,47 \\
\text { nto }\end{array}$ & 0,22 & $F_{(2,106)}=14,732$ & 0,000 & 0,226 & 2,578 & 0,011 \\
\hline \multicolumn{9}{|c|}{ Casadas com renda acima de dez salários mínimos } \\
\hline $\begin{array}{l}\text { Bem-estar } \\
\text { psicológico }\end{array}$ & $\begin{array}{l}\text { Poder } \\
\text { de decisão }\end{array}$ & 0,49 & 0,24 & $F_{(1,61)}=19,524$ & 0,000 & 0,492 & 4,419 & $<0,0001$ \\
\hline \multicolumn{9}{|c|}{ Casadas com renda abaixo de dez salários mínimos } \\
\hline \multirow[t]{2}{*}{$\begin{array}{l}\text { Bem-estar } \\
\text { psicológico }\end{array}$} & $\begin{array}{l}\text { Satisfação } \\
\text { com casame }\end{array}$ & $\begin{array}{l}0,31 \\
\text { tto }\end{array}$ & 0,10 & $F_{(1,44)}=4,614$ & 0,037 & 0,308 & 2,148 & 0,037 \\
\hline & $\begin{array}{l}\text { Poder de } \\
\text { decisão }\end{array}$ & 0,43 & 0,18 & $F_{(2,43)}=4,892$ & 0,012 & 0,301 & 2,185 & 0,034 \\
\hline \multicolumn{9}{|c|}{ Casadas, faixa etária 1 (de 24 a 38 anos) } \\
\hline \multirow[t]{2}{*}{$\begin{array}{l}\text { Bem-estar } \\
\text { psicológico }\end{array}$} & $\begin{array}{l}\text { Poder } \\
\text { de decisão }\end{array}$ & 0,37 & 0,13 & $F_{(1,53)}=8,316$ & 0,006 & 0,368 & 2,884 & 0,006 \\
\hline & $\begin{array}{l}\text { Satisfação } \\
\text { com casame }\end{array}$ & to 0,45 & 0,20 & $F_{(2,52)}=6,484$ & 0,003 & 0,268 & 2,039 & 0,047 \\
\hline \multicolumn{9}{|c|}{ Casadas, faixa etária 2 (de 39 a 60 anos) } \\
\hline $\begin{array}{l}\text { Bem-estar } \\
\text { psicológico }\end{array}$ & $\begin{array}{l}\text { Poder } \\
\text { de decisão }\end{array}$ & 0,46 & 0,21 & $F_{(1,52)}=14,277$ & 0,000 & 0,464 & 3,778 & $<0,0001$ \\
\hline
\end{tabular}


entre as variáveis. Dos pressupostos considerados por Tabachnick e Fidell (1996) para este tipo de análise, foram cumpridos integralmente os que se referem a necessidade de verificação da existência dos casos desviantes (outliers), do fenômeno de multicolinearidade (correlações bastante fortes entre as variáveis antecedentes que podem estar medindo a mesma coisa e que inflaciona o índice de variância) como também a verificação da presença de homocedasticidade (tendência dos desvios-padrão, de erros da predição serem aproximadamente equivalentes para todos os escores da variável critério). Quanto ao número de sujeitos, após ter sido feita uma análise preliminar, foi possível observar que apenas duas das variáveis antecedentes (poder de decisão e satisfação com o casamento) compuseram a regressão, sendo portanto suficientes o número de sujeitos. Na Tabela 1 podem ser visualizados os resultados da regressão múltipla para o bem-estar psicológico.

Os resultados encontrados para toda amostra tiveram como variável antecedente o fator de recompensa do trabalho denominado poder de decisão, contendo itens de autonomia, liberdade, trabalho estimulante e ajuda a outros, explicando sozinha $12 \%$ do bem-estar psicológico $\left(F_{(1,131)}=18,479 ; p<0,0001 ; \beta=0,352\right)$. É importante assinalar que nesta regressão não incluímos nas variáveis antecedentes a satisfação com casamento, pois parte da amostra não era casada e portanto não havia respondido ao questionário pertinente a esta variável.

Quando considerada a amostra de mulheres casadas, sem separar grupos de acordo com outras variáveis sóciodemográficas, duas variáveis antecedentes ou preditoras compuseram a equação de regressão - poder de decisão e satisfação com casamento - explicando um total de $22 \%$ de variância compartilhada do bem-estar psicológico. Das variáveis antecedentes, a que contribuiu mais foi o poder de decisão $\left(F_{(1,107)}=21,673 ; p<0,0001 ; \beta=0,410\right)$, explicando sozinha $17 \%$, enquanto a satisfação com o casamento contribui com $5 \%\left(F_{(2,106)}=14,732 ; p<0,0001, \beta=0,226\right)$.

Controlando-se estado civil e renda, algumas modificações puderam ser observadas. Para o grupo de mulheres casadas e com renda mensal acima de dez salários mínimos $^{3}$, a única variável que explicou o bem-estar psicológico foi poder de decisão $\left(F_{(1,61)}=19,524 ; p<0,0001\right.$, $\beta=0,492)$, explicando sozinha $24 \%$. Enquanto que para as mulheres casadas e com renda mensal abaixo de dez salários mínimos, fez parte como variável antecedente primeiro satisfação com casamento $\left(F_{(1,44)}=4,614 ; p<0,037 ; \beta=\right.$

\footnotetext{
${ }^{3} \mathrm{O}$ referencial de renda foi o salário mínimo, o qual no período da pesquisa era $R \$ 151,00$ (cento e cinqüenta e um reais), o equivalente a $\$ 84,00$ (oitenta e quatro dólares).
}

$0,308)$ explicando $10 \%$ e, em segundo, poder de decisão $\left(F_{(2,43)}=4,892 ; p<0,034 ; \beta=0,301\right)$, contribuindo com $8 \%$.

Quando controladas as variáveis estado civil e idade, observou-se que no grupo de mulheres mais jovens (faixa etária entre 24 e 38 anos) fizeram parte como variáveis antecedentes poder de decisão $\left(F_{(1,53)}=8,316 ; p<0,006 ; \beta\right.$ $=0,368)$, explicando $13 \%$ e a satisfação com casamento $\left(F_{(2,52)}=6,484 ; p<0,047 ; \beta=0,268\right)$, explicando $7 \%$, perfazendo um total de $20 \%$ em conjunto. Já as mulheres com faixa etária entre 39 e 60 anos tiveram uma única variável antecedente, poder de decisão, explicando $21 \%\left(F_{(1,52)}=\right.$ 14,277; $p<0,0001 ; \beta=0,464)$. Observando os resultados encontrados, é fácil notar que a variável que melhor explica o bem-estar psicológico neste estudo é poder de decisão, sendo que nos casos em que as variáveis sócio-demográficas foram controladas, em dois momentos, esta foi a única a explicar bem-estar psicológico.

\section{Discussão e Conclusão}

Os mitos e crenças que envolveram as conseqüências das atividades remuneradas das mulheres para sua saúde e bem-estar psicológico, e que criaram toda uma teoria de que estas deveriam permanecer em seus papéis tradicionais, vêm se diluindo a medida que as pesquisas realizadas a respeito da multiplicidade de papéis e bemestar psicológico têm encontrado níveis de bem-estar elevados entre mulheres que possuem um trabalho remunerado, em contraposição a níveis mais baixos em mulheres que não trabalham (Amatea \& Fong, 1991; Aston \& Lavery, 1993; Barnett \& Baruch, 1985; Barnett \& cols., 1992; Bullers, 1994; Gore \& Mangione, 1983; Gove \& Tudor, 1973; Hecht, 2001; Kandel, Davies \& Raveis, 1985; Kessler \& McRae, 1981; Kibria \& cols., 1990; Martire \& cols., 2000; Stephens \& cols., 1994; Vandewater \& cols., 1997; Verbrugge, 1983).

Embora as pesquisas científicas tenham apontado para ganhos em saúde física e mental (Vandewater \& cols., 1997; Verbrugge, 1983), os meios de comunicação continuam a mistificar as informações e a criar uma grande culpa para as mulheres ou casais que diferem do padrão estabelecido. $\mathrm{Na}$ verdade nossa sociedade não conseguiu ainda se desligar de um modelo que não funciona mais. Nos casamentos onde existe uma divisão mais igualitária das atividades domésticas e cuidado com os filhos, os ganhos são para todos, pois se a mãe diminui sua sobrecarga em casa e passa a contribuir financeiramente com seu trabalho, por outro lado o pai também sofre menos a pressão dos momentos de insegurança no emprego, e passa a conviver mais com os filhos criando um relacionamento mais estreito, o que 
tem favorecido ao bem-estar emocional dos filhos (Barnett \& Rivers, 1998).

São muitos os componentes que afetam o bem-estar psicológico, porém com relação a multiplicidade de papéis as abordagens têm sido feitas em termos de quantidade de energia que as pessoas dispõem na realização de suas atividades, no cumprimento das suas obrigações de papéis, e nos conflitos decorrentes destas atuações, como também têm sido consideradas as características sociais que são causadoras do distress psicológico.

Os resultados encontrados apontaram para um bom índice de bem-estar psicológico da amostra, com uma pontuação média de $1,86(d p=0,53)$ na escala do Questionário de Saúde Geral (QSG-12). As recompensas dos papéis avaliados foram mais elevadas do que as preocupações, o que confirma a noção de que mesmo desempenhando múltiplos papéis, o bem-estar psicológico é possível quando as recompensas são maiores que as preocupações (Sieber, 1974).

Pode-se observar ainda que os itens que as mulheres consideraram como mais recompensadores no papel do trabalho foram os que compuseram o fator poder de decisão (autonomia, estimulação, desafio, poder ajudar outros e ajuste do trabalho a necessidades e interesses). Este fator foi o que mais explicou o bem-estar psicológico encontrado na amostra, confirmando dados da literatura, e com um percentual de variância maior do que em pesquisas anteriores. Por exemplo, pesquisas como Aston e Lavery (1993) explicaram até 23\% de variância, Barnett e colaboradores (1992) explicaram no máximo 18\% de variância, Barnett e Marshall (1992) explicaram 12\%, Gore e Mangione (1983) explicaram 13\%, Kibria e colaboradores (1990) explicaram $24 \%$ e, nos estudos de Verbrugge (1983), o índice mais elevado de explicação chega aos $25 \%$, enquanto que o presente estudo explicou até $24 \%$, ou seja, o percentual de variância explicada pode ser considerado adequado aos padrões da literatura.

É interessante observar que no presente estudo, embora as recompensas do papel de mãe tenham sido as mais elevadas ( $m=3,63, \not p=0,40)$, elas não integraram a equação de regressão, não fazendo parte da explicação para o índice de bem-estar psicológico encontrado. Uma das possíveis explicações para respostas com um índice elevado de recompensas, pode estar na tendência em responder de acordo com o que é desejado socialmente, e o que se espera de uma boa mãe na nossa sociedade, é que ela perceba a maternidade como algo nobre e recompensador.

Para alguns autores, a explicação está no fato de que as crianças atuariam nas tensões constantes, como, por exemplo, aumentando as dificuldades econômicas, como também interferindo direta e indiretamente na qualidade do casamento, através da diminuição de tempo dos parceiros para dedicarem ao seu relacionamento, e através das tensões econômicas que podem causar, criando novas necessidades para a família (Brown \& Harris, 1978; Kandel \& cols., 1985). As conclusões na literatura são de que crianças não aumentam o bem-estar psicológico (Brown \& Harris, 1978; Kessler \& McRae, 1981).

Pesquisas (Gore \& Mangione, 1983; Kandel \& cols., 1985) têm encontrado um índice de bem-estar mais elevado entre os casados do que entre os não casados. Estudos como o de Pearlin e colaboradores (1981) comprovaram a importância de relacionamentos de qualidade como protetores contra os eventos estressantes, o que se sabe é que um bom casamento proporciona o senso de ser amado, estimado e valorizado como pessoa. Pessoas que se sentem amadas e valorizadas conseguem lidar com mais eficiência em momentos que se deparam com eventos indesejáveis, eventos estes que provocam um aumento no distress por promoverem mudanças e senso de perda de controle da situação. Por outro lado, situações de conflito nessas áreas podem ter um efeito devastador como elemento estressor, isto porque considera-se que conflitos interpessoais envolvam maior desgaste (Kandel \& cols., 1985; Tein, Sandler \& Zautra, 2000). Neste estudo, foram controladas as variáveis sóciodemográficas como estado civil, renda e idade para verificar as influências no bem-estar psicológico dos participantes.

Associações entre satisfação com o casamento e o bemestar psicológico também foram encontradas neste estudo. Interessante é observar como os resultados se apresentaram conjugados a outras variáveis sócio-demográficas. A satisfação com o casamento aparece como segunda preditora quando consideramos as mulheres casadas, passa a ser a primeira para o grupo de mulheres casadas e que possuem uma renda inferior a dez salários mínimos, atingindo $10 \%$ de explicação, e desaparece no grupo com renda superior a dez salários mínimos e no caso da faixa etária entre 39 e 60 anos. Sobre este aspecto podemos supor que mulheres que apresentam a satisfação com o casamento como principal preditora podem tanto dar maior importância ao casamento, por questões culturais, como podem não ter encontrado um trabalho que seja desafiador e estimulante que lhe proporcione as recompensas de um fator como poder de decisão. Sabe-se que desde muito jovens as mulheres são pouco encorajadas para desempenhar atividades que sugerem desafios, assim como desde muito jovens recebem mensagens de que não são tão competentes quanto os garotos, e isto influencia nas suas escolhas como também lhe é negado o poder de atuar nestes tipos de atividades (Barnett \& Rivers, 1998). 
O fator que mais contribuiu para explicar o índice de bem-estar psicológico foi o poder de decisão, ou seja, um fator que propicia o que muitos autores denominam de controle percebido (Amatea \& Fong, 1991; Barnett \& Marshall, 1992; Barnett \& cols., 1992; Bullers, 1994; Mirowsky \& Ross, 1989; Pearlin \& cols., 1981). Segundo estes autores, é o controle percebido que serve de mediador entre os papéis e o efeito para a saúde; é o trabalho pago que propicia o tipo de recompensas já comentadas anteriormente, e é capaz de proporcionar o aumento do controle percebido, já que desenvolve uma maior capacidade de lidar com situações novas e complexas, além de aumentar o seu acesso para fontes financeiras e sociais. Esta capacidade se estende por outras esferas de sua vida, permitindo então que a pessoa diante de estressores utilize técnicas de enfrentamento adequadas e encontre soluções para as situações difíceis.

É o controle percebido que favorece ao enriquecimento do Eu, promovendo uma auto-estima elevada, como também o senso de domínio do ambiente necessário, que lhe permite ver-se como um ser capaz de atuar sobre sua vida criando as condições, modificando e controlando-as, e como tivemos oportunidade de observar no processo do stress, descrito por Pearlin e colaboradores (1981), é de fundamental importância que o indivíduo mantenha o domínio e a auto-estima elevada quando estiver diante de situações difíceis, pois assim são capazes de visualizar a situação como algo que pode ser modificado por suas ações, podendo transformar situações adversas em oportunidades ou pelo menos podendo superá-las.

As mulheres que são capazes de se sustentar, ter um trabalho que crie novos caminhos, e não só a rotina do trabalho doméstico, são as que conseguem superar o sentimento de impotência que aparece presente quando a mesma se encontra diante de estressores. Estas mulheres não estão mais dependentes do poder econômico de seus maridos, e em momentos de dificuldades econômicas, de desemprego do companheiro, doenças ou crises no casamento, elas podem contar com o que o seu trabalho lhe proporciona como também com a rede de amigos criado pela sua participação no mercado de trabalho. Sem dúvida é reconhecido por diversos autores que o trabalho pago é o maior responsável aos benefícios proporcionados a saúde, isto porque os papéis não tradicionais implicam em um considerável ganho em poder o que torna possível sentimentos de independência, controle e positiva auto-estima, elementos essenciais a boa saúde (Rosenfield, 1980; Verbrugge, 1983). Quando os recursos financeiros da família são conseguidos pelo casal em proporções semelhantes, as outras atividades antes só desempenhadas pelas mulheres, passam também a ser desempenhadas pelo homem, tendo sido observado em algumas pesquisas que isto além de reduzir o distress das mulheres, não aumenta o distress para o homem (Mirowsky \& Ross, 1989).

Deve-se enfatizar que, para atuar em posições no mercado de trabalho, onde é possível ter autonomia nas atividades desempenhadas sem uma supervisão exaustiva, enfrentando situações desafiadoras, é necessário ter um nível de escolaridade elevado, pois este é um dos requisitos para se conseguir boas posições; no caso da amostra estudada a sua maioria apresentava um nível de escolaridade superior, o que pode ter contribuído indiretamente para o bem-estar psicológico. Esta variável também é considerada por autores como Mirowsky e Ross (1989) como capaz de contribuir diretamente sobre o bem-estar, pois a educação desenvolve no indivíduo a capacidade de solucionar problemas em várias esferas de sua vida, isto através do desenvolvimento de habilidades como leitura, escrita, capacidade de questionar e discutir, ou seja, ter ação e tomar decisões.

Desta maneira, como foi possível observar na descrição das características da amostra, a sua maioria possui um bom nível de instrução, como também a concentração de renda se encontra numa faixa que favorece para que estas mulheres possam ter trabalhos mais estimulantes e de autonomia em suas atividades e providenciar uma infra-estrutura mais adequada para que possam permanecer no mercado de trabalho. Portanto, não podemos esquecer que estas condições não estão presentes para a maioria das mulheres num país como Brasil, ficando nossos resultados limitados a faixa da população que possui as características da amostra, fazendo-se necessário futuros estudos na área considerando outras camadas populacionais. Porém, consideramos importante estudar a multiplicidade de papéis neste tipo de amostra, onde o desempenho de várias atividades não são confundidas com as condições inadequadas que o pessoal de baixa renda enfrenta.

Uma outra característica encontrada que confirma dados da Fundação Carlos Chagas (1998), é de que são as mulheres com nível superior as que mais conquistam espaço no mercado de trabalho formal, com a possibilidade de ter um trabalho com condições adequadas, favorecendo assim o seu bem-estar psicológico. Em estatísticas apresentadas por esta Fundação, o crescimento da atividade remunerada feminina no Brasil têm sido maior entre as que possuem maior tempo de escolarização, sendo que em 1998 na região Nordeste, $85 \%$ das mulheres que apresentavam uma escolaridade de 15 anos ou mais estavam economicamente ativas e estes percentuais não diferiram muito em outras regiões do país.

Em trabalho realizado por Leone (2000) a respeito do crescimento da renda familiar e do trabalho da mulher 
na região metropolitana de São Paulo entre os anos 1980 à 1990, a autora constatou que "foram as mulheres-chefes e cônjuges, com idade acima de 25 anos, escolarizadas, pertencentes a famílias sem nível de renda muito baixo, que mais aumentaram sua participação no trabalho remunerado" (Leone, 2000, p. 86). O que segundo a autora também pode ser verificado em outras regiões metropolitanas do Brasil, representando o fato de mais mulheres casadas terem começado a trabalhar por remuneração, como também das mais jovens não se afastarem do mercado de trabalho formal com a mudança do estado civil.

O presente estudo confirma dados da literatura com referência a importância do trabalho pago para o bemestar psicológico da mulher, e que a multiplicidade de papéis desempenhado pelas mulheres não é necessariamente sinônimo de distress, demonstrando assim que a teoria da acumulação de papéis, teoria da expansão (Sieber, 1974) se apresenta adequada como suporte para a explicação das conseqüências que envolvam a atuação em múltiplos papéis; assim como foram comprovadas as influências das características sociais no processo do stress, favorecendo assim para que mais estudos sejam feitos considerando estes aspectos.

Contudo, objetivando-se futuras pesquisas que envolvam a avaliação do bem-estar psicológico onde sejam utilizadas estas teorias, este trabalho pondera algumas sugestões que poderão auxiliar para o entendimento a respeito dos fatores que influenciam o bem-estar psicológico.

Primeiramente, devido a quantidade restrita de pesquisas nesta área em nossa população, seria adequado o desenvolvimento de pesquisas correlatas para que as comparações e implicações dos resultados possam se referir a parâmetros culturais semelhantes, enriquecendo a discussão.

É importante, também, que as próximas pesquisas utilizem algum instrumento que possa mensurar o suporte social recebido, que pudesse avaliar por exemplo, como é o apoio de familiares para os cuidados com os filhos, pois observou-se que a média de preocupação com os filhos não foi alta, o que pode ser talvez explicado por uma infraestrutura adequada nos cuidados com os mesmos.

Finalmente, outro aspecto a ser considerado é uma ênfase maior nas variáveis sócio-demográficas, já que confirmouse que as mesmas possuem um impacto considerável na explicação do índice de bem-estar, propiciando um maior poder comparativo entre as características sociais consideradas importantes no desencadeamento do processo do stress.

\section{Referências}

Almeida, D. M. \& Kessler, R. C. (1998). Everyday stressors and gender diferences in daily distress. Journal of Personality and Social Psychology, 75 (3), 670-680.

Amatea, E. S. \& Fong, M. L. (1991). The impact of roles stressors and personal resources on the stress experience of professional women. Psychology of Women Quarterly, 15, 419-430.

Aston, J. \& Lavery, J. (1993). The health of women in paid employment: Effects of quality of work role, social support and cynicism on psychological and physical well-being. Women and Health, 20, 1-25.

Barnett, R.C. \& Baruch, G.K. (1985). Women's involvement in multiple roles and psychological distress. Journal of Personality and Social Psychology, 49, 135-145.

Baruch, G. K. \& Barnett, R. C. (1986). Role quality, multiple role involvement and psychological well-being in midlife women. Journal of Personality and Social Psychology, 51, 578-585.

Barnett, R. C. \& Marshall, N. L. (1992). Worker and mother roles, spillover effects and psychological distress. Women and Health, 18, 9-40.

Barnett, R.C., Marshall, N.L. \& Sayer, A. (1992). Positive-spillover effects from job to home: A closer look. Women and Health, 19, 13-41.

Barnett, R. C. \& Rivers, C. (1998). She works/ he works: How two income families are happy, healthy, and thriving. USA: Harvard University Press.

Brown, G.W. \& Harris,T. (1978). Life-events and depression. Social origins of depression: A study of psycbiatric disorder in women. New York: The Free Press.

Bullers, S. (1994). Women's roles and health: The mediating effect of perceived control. Women and Health, 22, 11-30.

Dias, C. M. S. B. (1995). Relacionamento com filho adolescente: Percepção de mães. Tese de Doutorado Não-publicada. Instituto de Psicologia, Universidade de Brasília, DF.

Fundação Carlos Chagas (1998). Banco de dados sobre o trabalho das mulheres [On-line]. Disponível na Internet: http://www.fcc.org.br/pesquisa/ rgenero/bdtrabfm/

Goldberg, D. (1972). The detection of psychiatric illness by questionnaire. London, Oxford: University Press.

Goode, W. J. (1960). A theory of role strain. American Sociological Review, 25, 483-496.

Gore, S. \& Mangione, T. W. (1983). Social roles, sex roles and psychological distress: Additive and interactive models of sex differences. Journal of Health and Social Behavior, 24(12), 300-312.

Gove, W. R. \& Tudor, J. F. (1973). Adult sex roles and mental illness. American Journal of Sociology, 78, 812-835.

Hecht, L. M. (2001). Role conflict and role overload: Different concepts, different consequences. Sociological Inquiry, 71(1), 111-121.

Kandel, D. B., Davies, M. \& Raveis, V. H. (1985). The stressfulness of daily social roles for women: Marital, occupational and household roles. Journal of Health and Social Behavior, 26(3), 64-78.

Kessler, R. C. \& McRae Jr., J. A. (1981). Trends in the relationship between sex and psychological distress: 1957-1976. American Sociological Review, 46, 443-452.

Kibria, N., Barnett, R.C., Baruch, G. K., Marshall, N.L. \& Pleck, J.H. (1990). Homemaking-role quality and the psychological well-being and distress of employed women. Sex-Roles, 22, 327-347.

Lennon, M.C. (1994). Women, work, and well-being: The importance of work conditions. Journal of Health and Social Bebavior, 35, 235-247.

Leone, E. T. (2000). Renda familiar e trabalho da mulher na região metropolitana de São Paulo nos anos 80 e 90. Em M. I. B. Rocha (Org.), Trabalho e gênero (pp. 85 - 110). São Paulo: Editora 34.

Marks, S. R. (1977). Multiple roles and role strain: Some notes on human energy, time and commitment. American Sociological Review, 42, 921-936.

Martire, L. M., Stephens, M. A. P. \& Townsend, A. L. (2000). Centrality of women's multiple roles beneficial and detrimental consequences for psychological well-being. Psychology and Aging, 15(1), 148-156. 
Mirowsky, J. \& Ross, C. E. (1989). Social causes of psychological distress. NewYork: Aldine de Gruyter.

Nelson, D.L., Quick, J.C., Hitt, M.A. \& Moesel, D. (1990). Politics, lack of career progress, and work/home conflict: Stress and strain for working women. Sex-Roles, 23, 169-185.

Pearlin, L. I., Lieberman, M.A., Menaghan, E. G., \& Mullan, J.T. (1981). The stress process. Journal of Health and Social Behavior, 22, 337-356.

Pearlin, L. I. (1989). The sociological study of stress. Journal of Health and Social Behavior, 30, 241-256.

Possatti, I. C. \& Dias, M. R. (2001). Parâmetros psicométricos das escalas de qualidade dos papéis de mãe e do trabalho [Resumos]. Anais, II Congresso Norte Nordeste de Psicologia, (p.171). Salvador, Brasil.

Possatti, I. C., Dias, M. R. \& Gouveia, V.V. (2000). Versão abreviada do Questionário de Saúde Geral (QSG-12): Validação de construto [Resumos]. Anais, XXX Reunião Anual de Psicologia (p. 258). Brasília, Brasil.

Reifman, A., Biernat, M. \& Lang, E.L. (1991). Stress, social support, and health in married professional women with small children. Psychology of Women Quarterly, 15, 431-435.

Rosenfield, S. (1980). Sex differences in depression: Do women always have higher rates? Journal of Health and Social Behavior, 21, 33-42.

Stephens, M. A. P., Franks, M. M. \& Townsend, A. L. (1994). Stress and rewards in women's multiple roles: The case of women in the middle. Psychology and Aging, 9(1), 45-52.
Sieber, S. D. (1974). Toward a theory of role accumulation. American Sociological Review, 39, 567-578.

Tabachnick, B. G. \& Fidell, L. S. (1996). Using multivariate statistics ( $3^{\mathrm{a}}$ ed.). New York: Harper Collins.

Tein, J. Y., Sandler, I. N. \& Zautra, A. J. (2000). Stressful life events, psychological distress, coping and parenting of divorced mothers: A longitudinal study. Journal of Family Psychology, 14(1), 27-41.

Thoits, P. A. (1983). Multiple identities and psychological well-being: A reformulation and test of the social isolation hypothesis. American Sociological Review, 48, 174-187.

Vandewater, E. A., Ostrove, J. M. \& Stewart, A. J. (1997). Predicting women's well-being in midlife the importance of personality development and social role involvements. Journal of Personality and Social Psychology, 72(5), 1147-1160.

Verbrugge, L. M. (1983). Multiple roles and physical health of women and men. Journal of Health and Social Behavior, 24(3), 16-30.

Sobre os autores

Izabel Cristina Possatti é Mestre em Psicologia Social pela Universidade Federal da Paraíba. Professora do Depto de Psicologia da UFPB.

Mardônio Rique Dias é Doutor em Psicologia Social pela Universidade de Brasília. Professor Adjunto do Depto e do Mestrado em Psicologia Social da UFPB. Coordenador do Núcleo de pesquisa "Aspectos Psicossociais de Prevenção e da Saúde Coletiva". 